\title{
Prediction of Residual Stress Field in Milling Planes by Establishing Bivariate Mathematical Models
}

\author{
Fengyun Wang ${ }^{1, a}$, Kuanmin Mao ${ }^{1, a}$, Shanguo $\mathrm{Wu}^{2}$, Yikang $\mathrm{Du}^{1}$ and Xiaobo Mao ${ }^{1}$ \\ ${ }^{1}$ School of Mechanical Science and Engineering, Huazhong University of Science and Technology, \\ Wuhan 430074, China \\ ${ }^{2}$ Yantai Chengxin Steel Grating Co. Ltd, Yantai 264006, China
}

\begin{abstract}
The knowledge of the distribution of residual stresses in milling planes is of paramount importance to understand the surface qualities of engineering components, research on manufacturing processes and improve the assembly qualities of machines. For this purpose, a prediction method for the residual stress fields was proposed. Residual stresses of some surface discrete points of a rectangular specimen were measured. Based on the measurements, the residual stress field was predicted by establishing bivariate mathematical models. The obtained results are close to the experimental technique and superior to the previous average method. The predicted stress fields are almost symmetrical along the length or width. The proposed method avoids simulating the process of residual stresses generation and has a unique potential application for engineering components of which the generation process of residual stresses can hardly be simulated in detail.
\end{abstract}

\section{Introduction}

Residual stresses are special self-equilibrium stresses remained within engineering with no thermal gradients or external forces. They can influence the mechanical properties of engineering components such as the distortion, structural stability and fatigue life [1]. The surface residual stress, as a critical parameter of evaluating the surface quality and assembly quality, can directly affect the working performance and working life [2-3]. So for design, manufacturing and structural integrity assessment, it is necessary to know the residual stress distribution of surface residual stress as far as possible.

Residual stresses are inevitably existed in processes such as manufacturing, remanufacturing and using of mechanical components. The process of residual stresses generation is hard to be simulated. To date, surface residual stress could be measured by experimental methods[4-8] such as X-ray diffraction (XRD). However, due to the time consuming, expensive and limits of testing on some discrete points, the measured stress could only reflect the local stress characteristics. It is hard to find out the distribution of residual stress when the machine part is bigger just using experimental methods. Previous studies have ignored the initial residual stresses in the finite element analysis, considered initial residual stresses using average stress or just researched on the distribution of residual stresses in a straight line recently [9-11]. However, little information has been focused on establishing bivariate

\footnotetext{
a Corresponding author : kmmao4546@sina.com, fywang0213@sina.com
} 
regression mathematical functions to predict plane residual stress fields.

The purpose of this paper is to obtain the distribution of residual stresses in a milling surface of 316 stainless steel shown in Figure.1 by establishing bivariate mathematical models. Based on the theoretical analysis of surface residual stress, the prediction method is first described. Furthermore, the computational procedure is shown. In addition, the predicted residual stresses of the obtained mathematical models are compared not only with the experimental data but also with the mean. Finally, the obtained residual stress field is analyzed. With the help, it would be easy to know the distribution of surface residual stress field and has a better understanding of the surface qualities of engineering components.

\section{Methodology}

\subsection{Theoretical background}

According to the elastic-plasticity theory, the stress components satisfy the equilibrium equations and boundary condition as follows:

$$
\begin{gathered}
\partial \sigma_{x} / \partial x+\partial \tau_{x y} / \partial y+\partial \tau_{x z} / \partial z+f_{x}=0, \partial \sigma_{y} / \partial y+\partial \tau_{x y} / \partial x+\partial \tau_{y z} / \partial z+f_{y}=0 \text { and } \partial \sigma_{z} / \partial z+\partial \tau_{y z} / \partial y+\partial \tau_{x z} / \partial x+f_{z}=0 \\
l \sigma_{x}+m \tau_{x y}+n \tau_{x z}=\overline{f_{x}}, l \tau_{x y}+m \sigma_{y}+n \tau_{y z}=\overline{f_{y}} \text { and } l \tau_{z x}+m \tau_{y z}+n \sigma_{z}=\overline{f_{z}}
\end{gathered}
$$

where $f_{x}, f_{y}$ and $f_{z}$ represent the body force, $\bar{f} x, \bar{f} y$ and $\bar{f} z$ denote the surface force, $\sigma_{x}, \sigma_{y}$ and $\sigma_{z}$ are the residual stress component in the $x, y$ and $z$ directions, respectivelly; $l, m$ and $n$ denote the three directions cosine of the normal to the boundary plane; $\tau_{x y}, \tau_{y z}$ and $\tau_{x z}$ are the shear stress on the $x y, y z$ and $x z$ planes, respectively.

Residual stresses are special stresses of a mechanical component when the surface forces are zero. In the plane parallel to the $x-y$ coordinate plane, $l=m=0$ and $n=1$. Thus, it could be concluded that $\sigma_{z}=\tau_{x z}=\tau_{y z}=0$ from (2). Furthermore, (1) could be deduced to the follows:

$$
\partial \sigma_{x} / \partial x+\partial \tau_{x y} / \partial y+f_{x}=0 \text { and } \partial \sigma_{y} / \partial y+\partial \tau_{x y} / \partial x+f_{y}=0
$$

Ignoring the body forces, the solution of the differential equations (3) is $\sigma x=\partial^{2} \varphi / \partial^{2} y, \sigma y=\partial^{2} \varphi / \partial^{2} x$ and $\tau_{x y}=-\partial^{2} \varphi / \partial x \partial y$. No matter what the function $\varphi$ is, the three stress components could satisfy (3). The three stress components could be described as functions of $x$ and $y$ as follows:

$$
\sigma_{x}=f_{x}(x, y), \sigma_{y}=f_{y}(x, y) \text { and } \tau_{x y}=f_{x y}(x, y)
$$

\subsection{Description of the proposed method}

According to (4), the three residual stress components could be described as the function of two planar natural coordinates. Thus, bivariate mathematical functions for predicting residual stress components could be established using measurements of surface points. To obtain the suitable mathematical functions, residual stress components of some points on the surface could be first measured and then be used to establish or check the bivariate mathematical functions.

\section{Experimental procedures and results}

\subsection{Sample and experimental setup}


The experimentation was performed on a rectangular specimen. The geometry of the specimen and the test instrument are shown in Figure 1. The dimensions of the specimen are: length $=30 \mathrm{~mm}$, width $=10 \mathrm{~mm}$, and height $=5 \mathrm{~mm}$. The material of the sample is 316 stainless steel. Residual stresses were tested by using $X R D$ technique. The test instrument used was the Proto XRD residual stress test instrument.

To obtain the stress components $\sigma_{x}, \sigma_{y}$ and $\tau_{x y}$, measurements were made with Mn_K-Alpha radiation in the length, width and rotation $45^{\circ}$ direction in the gage region limited by a collimator diameter of $1 \mathrm{~mm}$. The $\sin ^{2} \psi$ method was used with seven beta angles at maximum beta angle interval from $-25^{\circ}$ to $25^{\circ}$. The hkl-311 diffracting planes were selected. The bragg angle was $152.8^{\circ}$.

Because it is rather difficult to test the residual stresses of the points on the boundary line accurately, eleven points on the upper surface were measured as shown in Figure 1. The origin of the coordinate system was located at the center of the surface. The x-axis was along the length direction while the y-axis was along the width direction. The 1 st to 9 th points were the quadrant points that divided the length and width directions into four equal parts. Meanwhile, the coordinates of the 10th and 11 th points were $(-11,25,3.75)$ and $(-3.75,1.25)$, respectively.

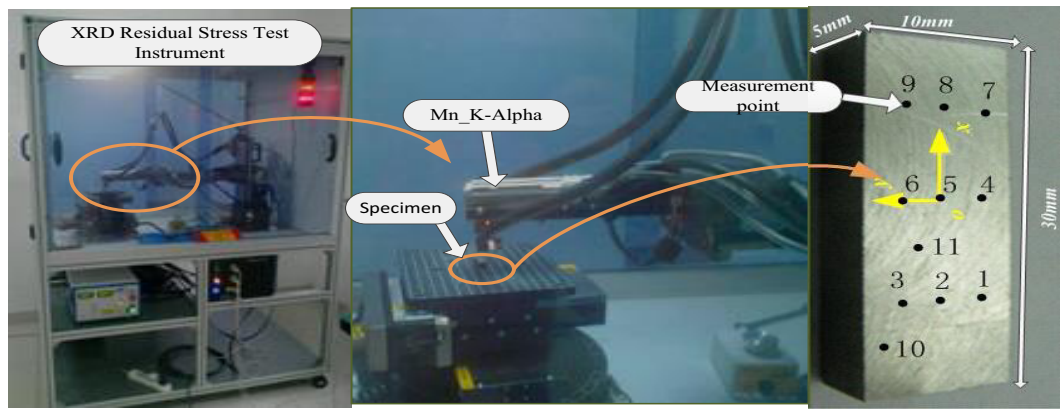

Figure 1. Experimental setup and test points.

\subsection{Experimental results}

The obtained residual stresses of the 11 test points are shown in Figure 2. The maximum stress and minimum stress of the longitudinal residual stress $\sigma_{x}$, transversal residual stress $\sigma_{y}$ and shear stress $\tau_{x y}$ are $252.50 \mathrm{MPa}$ and $-77.59 \mathrm{MPa}, 796.27 \mathrm{MPa}$ and $513.04 \mathrm{MPa},-49.50 \mathrm{MPa}$ and $-268.59 \mathrm{MPa}$, respectively. It can be seen that the fluctuation ranges are large. Meanwhile, the means of the three stress components are $61.77 \mathrm{MPa}, 623.42 \mathrm{MPa},-169.11 \mathrm{MPa}$, respectively.

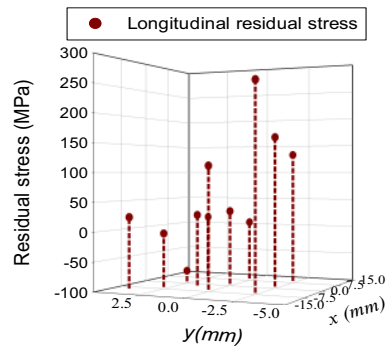

(a)

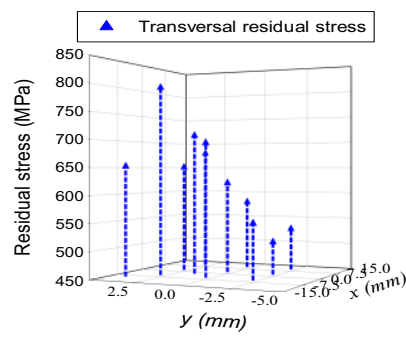

(b)

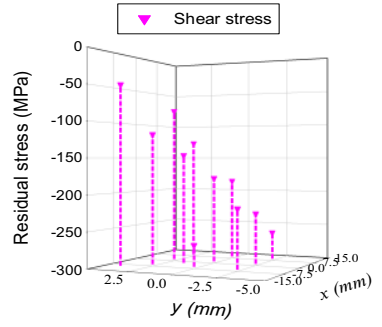

(c)

Figure 2. Experiments of (a) $\sigma x$, (b) $\sigma_{y}$, and (c) $\tau_{x y}$ of 11 test points. 


\subsection{Choice of modelling points and checking points}

To find suitable mathematical models for predicting the residual stress field, some points would be used to establish mathematical models, while another would be used to check the obtained models. These points are shown in Figure 3. The 1st to 9th points were the modelling points to establish the mathematical models. Meanwhile, the 10th and 11th points were the checking points to validate the models.

Similarly to residual stresses in the planes parallel to the $x-y$ coordinate plane, some of residual stress components of points in planes parallel to the $x-y$ or $y-z$ coordinate plane could also be deduced. The stress components $\sigma_{y}=\tau_{x y}=\tau_{y z}=0$ in the planes parallel to the $x$-z plane, while $\sigma_{x=} \tau_{x y}=\tau_{x z}=0$ in the planes parallel to the $y-z$ plane. Thus, in the $x-y$ plane, the stress components $\sigma_{y}=\tau_{x y}=0$ on the boundary lines parallel to the $x$-axis, while both of the $\sigma_{x}=\tau_{x y}=0$ on the boundary lines parallel to the $y$-axis.

To avoid checking residual stresses of points on boundary lines and improve the prediction accuracy, some points on the boundary lines could be selected to establish the mathematical models. The selected boundary points of the three residual stress components are shown in Figure 3 . Ten points on boundary lines parallel to the $y$-axis, ten points on boundary lines parallel to the $x$-axis and sixteen points on boundary lines parallel to the $x$-axis or $y$-axis were used to establish the model of $\sigma_{x}$, $\sigma_{y}$ and $\tau_{x y}$ respectively.

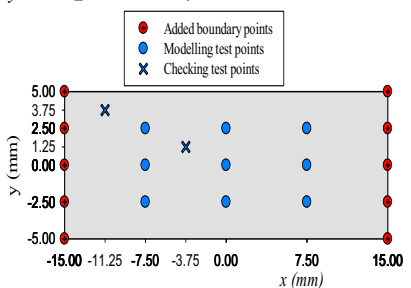

(a)

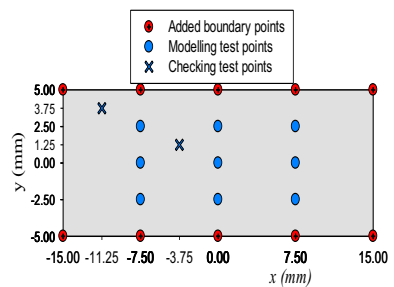

(b)

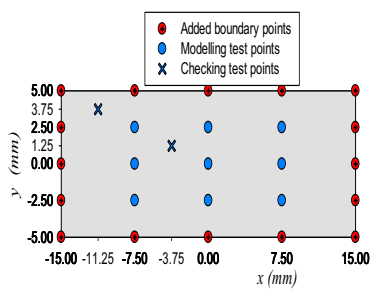

(c)

Figure 3. Choice of points to establish or validate models of (a) $\sigma_{x}$, (b) $\sigma_{y}$ and (c) $\tau_{x y}$.

\subsection{Establishment of bivariate mathematical models}

Based on the test points and boundary points, An automatic curve fitting and equation estimating software Table Curve 3D was used to find suitable bivariate mathematical models. By importing the data $\left(\mathrm{x}, \mathrm{y}, \sigma_{x}\right),\left(\mathrm{x}, \mathrm{y}, \sigma_{y}\right)$ and $\left(\mathrm{x}, \mathrm{y}, \tau_{\mathrm{xy}}\right)$ of the mentioned modeling points into Tablecurve 3D v4.0.01 software, respectively, the obtained data graphs were shown in Figure 4. Each graph contains a 3D scatter plot or interpolated surface of the currently selected XYZ data.

Usually functions with bigger coefficients of determination $r^{2}$ have better agreement between predicted and measured capillary pressure curves. According to the sequence of coefficients of determination $\mathrm{r}^{2}$ from large to small, it was found that surface-fit graphs of Chebyshev X, Y Bivariate Polynomials Order 4 shown in Figure 5 were similar with graphs in Figure 4 . The coefficients of determination $\mathrm{r}^{2}$ of the three stress components $\sigma \mathrm{x}, \sigma \mathrm{y}$ and $\tau \mathrm{xy}$ were $0.98507968,0.99426621$ and 0.91892214, respectively. Thus, the following Chebyshev $X, Y$ Bivariate Polynomials Order 4 was chosen as the mathematical model of the surface residual stress components:

$$
\begin{aligned}
f(x, y)= & a+b T_{1}\left(x^{\prime}\right)+c T_{1}\left(y^{\prime}\right)+d T_{2}\left(x^{\prime}\right)+e T_{1}\left(x^{\prime}\right) T_{1}\left(y^{\prime}\right)+f T_{2}\left(y^{\prime}\right)+g T_{3}\left(x^{\prime}\right)+h T_{2}\left(x^{\prime}\right) T_{1}\left(y^{\prime}\right)+i T_{1}\left(x^{\prime}\right) T_{2}\left(y^{\prime}\right) \\
& +j T_{3}\left(y^{\prime}\right)+k T_{4}\left(x^{\prime}\right)+l T_{3}\left(x^{\prime}\right) T_{3}\left(y^{\prime}\right)+m T_{2}\left(x^{\prime}\right) T_{2}\left(y^{\prime}\right)+n T_{1}\left(x^{\prime}\right) T_{3}\left(y^{\prime}\right)+o T_{4}\left(y^{\prime}\right)
\end{aligned}
$$

where $x^{\prime}=x / 15, y^{\prime}=y / 5, \mathrm{~T}_{n}\left(x^{\prime}\right)=\cos \left(n \cdot \operatorname{acos}\left(x^{\prime}\right)\right), \mathrm{T}_{n}\left(y^{\prime}\right)=\cos \left(n \cdot \operatorname{acos}\left(y^{\prime}\right)\right), f(x, y)$ denotes the residual stress component $\sigma_{x}, \sigma_{y}$ or $\tau_{x y}$, and $a-o$ are the fitting coefficients. The values of the coefficients $a-o$ in (5) are listed in Table 1. 


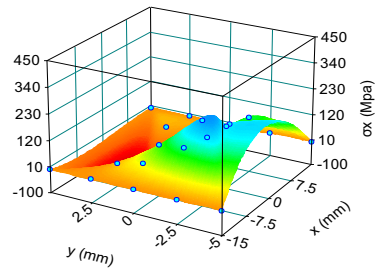

(a)

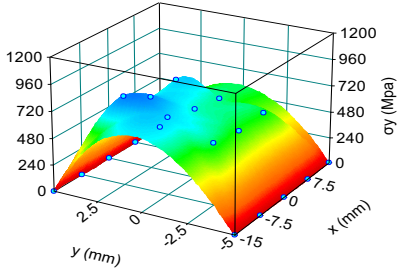

(b)

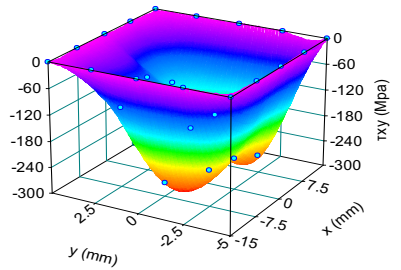

(c)

Figure 4. Data graphs of (a) $\sigma_{x}$, (b) $\sigma_{y}$ and (c) $\tau_{x y}$ obtained by importing the coordinate and stress components of mentioned modeling points into Tablecurve $3 \mathrm{D}$ v4.0.01 software.

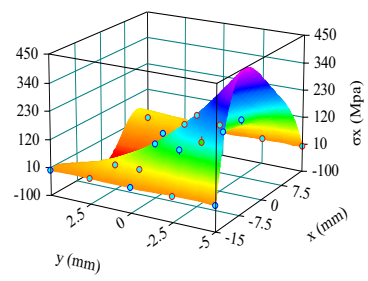

(a)

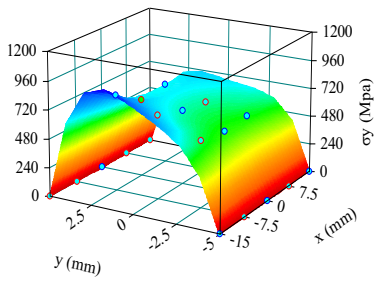

(b)

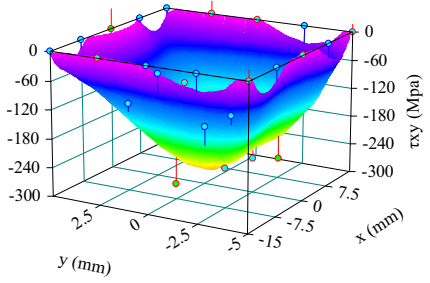

(c)

Figure 5. Surface-fit graphs of (a) $\sigma_{x}$, (b) $\sigma_{y}$ and (c) $\tau_{x y}$ displaying the importing data along with the surface for the fitted Chebyshev X, Y Bivariate Polynomials Order 4.

Table 1. Coefficients of residual stress model in (5).

\begin{tabular}{|c|c|c|c|}
\hline Coefficient & Value in $\sigma_{x}$ & Value in $\sigma_{y}$ & Value in $\tau_{x y}$ \\
\hline $\mathrm{a}$ & 37.385844 & 178.49217 & 10.088706 \\
\hline $\mathrm{b}$ & 10.719248 & 16.697397 & 0.2395292 \\
\hline $\mathrm{c}$ & 43.101709 & 23.707313 & 1.3812165 \\
\hline $\mathrm{d}$ & 18.446365 & 17.984915 & 6.1213734 \\
\hline $\mathrm{e}$ & 16.6463 & 10.533959 & 0.1313418 \\
\hline $\mathrm{f}$ & 14.241578 & 138.78497 & 7.9157676 \\
\hline $\mathrm{g}$ & 10.719248 & 0.1822267 & 0.2395292 \\
\hline $\mathrm{h}$ & 42.995814 & 0.4381588 & 0.8206999 \\
\hline $\mathrm{i}$ & 0.6049351 & 16.879624 & 0.3774382 \\
\hline $\mathrm{j}$ & 0.1058953 & 23.707313 & 1.3812165 \\
\hline $\mathrm{k}$ & 18.548416 & 1.153549 & 3.6882823 \\
\hline $\mathrm{l}$ & 16.6463 & 0 & 0.0938155 \\
\hline $\mathrm{m}$ & 13.654984 & 17.292786 & 7.5745897 \\
\hline $\mathrm{n}$ & $3.25 \mathrm{E}-19$ & 10.533959 & 0.0938155 \\
\hline $\mathrm{o}$ & 0.9776567 & 39.24578 & 0.6976252 \\
\hline
\end{tabular}

\section{Results and discussion}

\subsection{Comparison of experiments, mean and predicted stresses}

Figure 6 displays the comparison of experiments of the mentioned 11 test points, mean of experiments and predicted stresses of the obtained mathematical models. The solid lines are composed of experiments, while the dashed lines are of the means and the predicted stresses. It can be noted that the means have a significant difference with experiments. The differences between them are bigger than $40 \mathrm{MPa}$ and the maximum difference is up to nearly 200MPa. However, the predicted stresses are close to experiments, and the differences between them are basically less than 20Mpa. Thus, the proposed method is superior to the average method. 


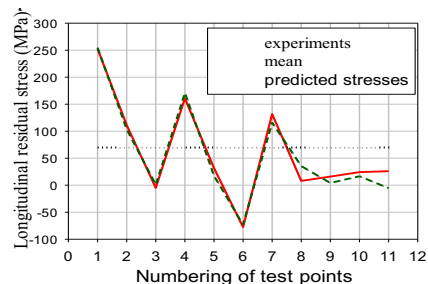

(a)

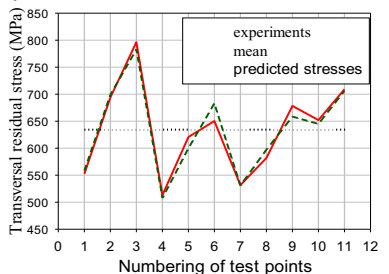

(b)

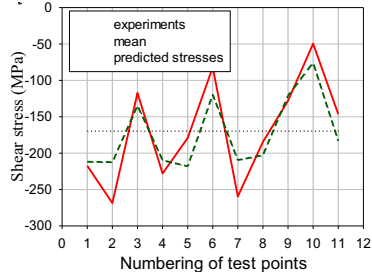

(c)

Figure 6. Comparison of experiments, mean of eperiments and predicted stresses of (a) $\sigma_{x}$, (b) $\sigma_{y}$ and (c) $\tau_{x y}$.

\subsection{Analysis of predicted residual stress field}

The predicted residual stress fields of the obtained mathematical models are shown in Figure 5. It can be seen that the stress fields are almost symmetrical along the width or length. The longitudinal residual stress $\sigma_{x}$ is symmetrical along the length, the transversal residual stress $\sigma_{y}$ is symmetrical along the width, and the shear stress $\tau_{x y}$ is symmetrical along the length and width. Meanwhile, the maximum stress or minimum stress exists in the symmetry axis or the boundary lines. The maximum stresses of $\sigma_{x}, \sigma_{y}$ and $\tau_{x y}$ are near $450 \mathrm{MPa}, 1200 \mathrm{MPa}$ and $0 \mathrm{MPa}$, while the minimum stresses are near $100 \mathrm{MPa}, 0 \mathrm{MPa}$ and $-300 \mathrm{MPa}$. Fluctuation ranges are about $550 \mathrm{MPa}, 1200 \mathrm{MPa}$ and $300 \mathrm{MPa}$. It indicates that it is of supreme importance to explore such a method to establish a mathematical model to predict the surface residual stress field.

\section{Conclusions}

To reconstruct the milling surface residual stress field, a prediction method by establishing bivariate mathematical functions based on measurements and boundary points is proposed. The results show that the prediction errors are basically less than 20Mpa and the distributions of the three stress components are almost symmetrical along the length or width. The proposed method is favorable to a significant underestimation of the residual stress field of milling surface. The Chebyshev X, Y Bivariate Polynomials Order 4 was the suitable mathematical model in the specimen. Whether there is a unified function that could display residual stress fields of different materials and structures is worthy to be studied.

\section{Acknowledgements}

This work was supported by the High-End CNC Machine Tools and Basic Manufacturing Equipment Technology Major Projects (Grant no. 2013ZX04005-011).

\section{References}

1. I. Samardzic, T. Vuherer, D. Maric, P. Konjatic, Metalurgija. 54, 527 (2015).

2. F. Gunnberg, M. Escursell, M. Jacobson, J. Mater. Process. Tech. 174, 82 (2006).

3. M. Liu, J.-i. Takagi, A. Tsukuda, J. Mater. Process. Tech. 150, 234 (2004).

4. M. Birkholz, C. Genzel, T. Jung, J. Appl. Phys. 96, 7202 (2004)

5. G. S. Schajer, Exp. Mech. 50, 245 (2010).

6. M. Ya, F. L. Dai, H. M. Xie, J. Lu, Acta. Mech. Sinica. 19, 567 (2003).

7. Y. Y. Santana, J. G. La Barbera-Sosa, M. H. Staia, Surf. Coat. Technol. 201, 2092 (2006).

8. M. Ya, P. Marquette, F. Belahcene, J. Lu, Mater. Sci. Eng. A. 382, 257 (2004).

9. M. Achintha, D. Nowell, K. Shapiro, P. J. Withers, Surf. Coat. Technol. 216, 68 (2013).

10. A. M. Korsunsky, G. M. Regino, J . Appl. Mech-T. Asme. 74, 717 (2007).

11. A. T. DeWald, M. R. Hill, Exp. Mech. 46, 473 (2006). 